\title{
Spatio-temporal anomalous diffusion in heterogeneous media by nuclear magnetic resonance
}

Cite as: J. Chem. Phys. 135, 034504 (2011); https://doi.org/10.1063/1.3610367

Submitted: 21 March 2011 . Accepted: 23 June 2011 . Published Online: 20 July 2011

M. Palombo, A. Gabrielli, S. De Santis, C. Cametti, G. Ruocco, and S. Capuani

ARTICLES YOU MAY BE INTERESTED IN

Anomalous diffusion in heterogeneous porous media

The Physics of Fluids 31, 965 (1988); https://doi.org/10.1063/1.866716

Spin Diffusion Measurements: Spin Echoes in the Presence of a Time-Dependent Field Gradient

The Journal of Chemical Physics 42, 288 (1965); https://doi.org/10.1063/1.1695690

Spin echo of spins diffusing in a bounded medium

The Journal of Chemical Physics 60, 4508 (1974); https://doi.org/10.1063/1.1680931

The Journal

of Chemical Physics 2018 EDITORS' CHOICE 


\title{
Spatio-temporal anomalous diffusion in heterogeneous media by nuclear magnetic resonance
}

\author{
M. Palombo, ${ }^{1,2}$ A. Gabrielli, ${ }^{1,3}$ S. De Santis, ${ }^{1,2}$ C. Cametti, ${ }^{1}$ G. Ruocco,,${ }^{1,2}$ \\ and S. Capuani ${ }^{1,2, a)}$ \\ ${ }^{1}$ Physics Department, Sapienza University of Rome, P.le A.Moro, 500185 Rome, Italy \\ ${ }^{2}$ CNR IPCF UOS Roma, Physics Department, Sapienza University of Rome, P.le A.Moro, 500185 Rome, Italy \\ ${ }^{3}$ ISC-CNR, Via dei Taurini, 1900185 Rome, Italy
}

(Received 21 March 2011; accepted 23 June 2011; published online 20 July 2011)

\begin{abstract}
In this paper, we describe nuclear magnetic resonance measurements of water diffusion in highly confined and heterogeneous colloidal systems using an anomalous diffusion model. For the first time, temporal and spatial fractional exponents, $\alpha$ and $\mu$, introduced within the framework of continuous time random walk, are simultaneously measured by pulsed gradient spin-echo NMR technique in samples of micro-beads dispersed in aqueous solution. In order to mimic media with low and high level of disorder, mono-dispersed and poly-dispersed samples are used. We find that the exponent $\alpha$ depends on the disorder degree of the system. Conversely, the exponent $\mu$ depends on both bead sizes and magnetic susceptibility differences within samples. The new procedure proposed here may be a useful tool to probe porous materials and microstructural features of biological tissue. (C 2011 American Institute of Physics. [doi:10.1063/1.3610367]
\end{abstract}

\section{INTRODUCTION}

In the last few decades, nuclear magnetic resonance (NMR) diffusion measurements of water molecules have been a topic of extensive research, having broad applications across a number of disciplines ranging from material science to biophysics ${ }^{1}$ and medicine. ${ }^{2}$ Translational self-diffusion in liquid systems can be measured using pulse field gradient techniques, by applying magnetic gradient pulses (named diffusion gradients) to the system, in addition to the static magnetic field of the instrument itself. The signal attenuation, that depends on both diffusion gradient strength and diffusion time, is simply the Fourier transform (FT) of the average motion propagator (MP). When the MP is Gaussian, NMR signal attenuation follows a mono-exponential Stejskal-Tanner decay. ${ }^{3}$ Conversely, when the motion is described by a nonGaussian propagator, the signal attenuation deviates from a mono-exponential decay. ${ }^{4,5}$

Up to now, restricted diffusion methods, based on Gaussian diffusion approximation, have been the most popular and commonly accepted NMR methods to investigate heterogeneous media. In particular, when measured in a porous system, apparent diffusion coefficient , as a function of time, ${ }^{6-9}$ provides information about pore structure by means of surface to volume ratio and tortuosity. Moreover, the q-space diffusion diffraction studies ${ }^{10,11}$ and their recent developments ${ }^{12,13}$ provide geometrical microstructural characterization and information on pore size of heterogeneous media. In general, all the above methods require a priori knowledge (partial or deeper, depending on the method) about the media under investigation. A more sophisticated technique, based on quantum field perturbation theory and valid in the limit of small

\footnotetext{
a) Author to whom correspondence should be addressed. Electronic mail: silvia.capuani@roma1.infn.it.
}

amplitude and small correlation length of disorder, has been recently introduced by Novikov and Kiselev. ${ }^{14}$

In many practical situations, the dynamics of water in porous media is restricted by confining boundaries. Crowding, caging, and geometrical traps may lead to departures from Gaussian diffusion. Here, we propose a different approach to obtain microstructural information from heterogeneous media by means of diffusion NMR methods based on non-Gaussian diffusion theory. It is well known that Gaussian diffusion predicts a linear relation between mean square displacement (MSD) of diffusing particles and the time during which the diffusion occurs. Conversely, anomalous diffusion (AD) is characterized by a MSD of diffusing particles growing nonlinearly in time, i.e., $\left\langle[x(t)-x(0)]^{2}\right\rangle \propto t^{v}$ (with $v \neq 1$ ). This is a property of many complex systems and their related phenomena have been observed in various physical and scientific fields. ${ }^{15-20}$

In this paper, we use the continuous time random walk (CTRW) model developed by Metzler and Klafter, ${ }^{21}$ as an effective approach to describe the features of AD. According to these authors, molecular AD in media can be depicted by an effective approach for which the MP is described as the solution of fractional diffusion equations, arising from the CTRW model. These equations involve two fractional exponents, $\alpha$ and $\mu$, which are the orders of the time and space fractional derivatives, respectively. ${ }^{22}$ The theoretical framework of CTRW is well established and has been corroborated by huge amounts of Monte Carlo simulations (see, for example, Ref. 23) together with several experimental studies, mainly obtained by using fluorescent spectroscopy. ${ }^{24,25}$ However, to our knowledge, an experimental $\alpha$ vs $\mu$ phase diagram, showing the competition between superdiffusion and subdiffusion of diffusing molecules in heterogeneous media, has never been carried out. Since NMR signal is 
obtained from an ensemble of spins as the FT of the averaged MP, in our opinion NMR techniques are the best experimental procedures to test the validity of such an effective theory.

In this paper, we have experimentally measured the effective fractional exponents $\alpha$ and $\mu$ by means of NMR methods, based on pulsed gradient spin-echo (PGSE) technique, ${ }^{3}$ and have provided an evidence of their interplay for the first time.

As it will be explained in Sec. II, in the present study, we use an $\mathrm{AD}$ model to describe the behavior of water molecules in porous media characterized by packed micro-beads polydispersed in aqueous solution. In Sec. III, materials and methods, used to investigate spatio-temporal anomalous diffusion in heterogeneous media, are described. PGSE NMR experiments were performed in controlled porous media characterized by packed micro-beads of various sizes, mono- and poly-dispersed in aqueous solution. Mono-dispersed (monod) and poly-dispersed (poly-d) samples were used to check the potential ability of the exponents $\alpha$ and $\mu$ to detect different degrees of system disorder (Sec. III A). Fractional exponent $\alpha$ was measured by collecting the PGSE signal attenuation as a function of diffusion times compared to the asymptotic expression of FT of the MP for subdiffusive regime, obtained from Metzler's review. ${ }^{21}$ Moreover, the exponent $\mu$ was extracted by fitting the expression of FT of the MP for superdiffusive regime, obtained again from Metzler's review, ${ }^{21}$ to the PGSE signal attenuation as a function of gradients strength. Because the internal background gradient effect in porous media usually plays an important role in microstructural data interpretation, ${ }^{26}$ internal magnetic field gradient $\left(G_{i n t}\right)$ measurements were performed to investigate the influence of the magnetic susceptibility differences $\left(\Delta \chi_{m}\right)$ on measured $\alpha$ and $\mu$ parameters (Sec. III B). In Sec. IV, experimental results are reported and discussed. Our experimental results demonstrate that the exponent $\alpha$ strongly depends on the disorder degree of the system, and it is independent of $\Delta \chi_{m}$ (Secs. IV A and IV B). Conversely, the exponent $\mu$ is strongly correlated with $\Delta \chi_{m}$ and pore sizes (Secs. IV B and IV C). In summary, mono-d samples are characterized by both ordinary diffusion and spurious magnetic susceptibility effect (Sec. IV C), while poly-d samples show a real effective subdiffusion of water molecules in a significative spatio-temporal range.

\section{THEORY}

Taking into account the CTRW model, ${ }^{21}$ each molecular displacement in a diffusion process can be characterized by two parameters: a waiting time $\tau$ elapsing between two consecutive steps, and a variable displacement length $\xi$. The quantities $\tau$ and $\xi$ are usually assumed as independent random variables, distributed according to the probability densities $\psi(\tau)$ and $\lambda(\xi)$. When $\psi(\tau) \simeq \tau^{-1-\sigma}$, with $0<\sigma<1$, and $\lambda(\xi)$ is characterized by a finite variance, the characteristic waiting time $T=\int_{0}^{\infty} \tau \psi(\tau) d \tau$ diverges and the resulting CTRW is subdiffusive. As a consequence, the asymptotic behaviors of the FT of the MP is characterized by

$$
\begin{aligned}
& W(q, t) \simeq\left\{e^{-K_{\alpha} q^{2} t^{\alpha}}, \quad \text { when } q^{2} \ll \frac{1}{K_{\alpha} t^{\alpha}},\right. \\
& \frac{1}{K_{\alpha} q^{2} t^{\alpha}}, \quad \text { when } q^{2} \gg \frac{1}{K_{\alpha} t^{\alpha}},
\end{aligned}
$$

where $\alpha=\sigma$ (if $\sigma \geq 1, \alpha=1$ ). Moreover, $K_{\alpha}$ is a generalized diffusion constant. Under these conditions, the MSD grows sublinearly in time, namely $\left\langle x^{2}(t)\right\rangle \simeq t^{\alpha}$. Vice versa, when $\lambda(\xi) \simeq|\xi|^{-1-\rho}$, with $0<\rho<2$, and $\psi(\tau)$ is characterized by a finite mean value, the jump length variance $\Sigma^{2}$ $=\int_{-\infty}^{\infty} \xi^{2} \lambda(\xi) d \xi$ diverges and a superdiffusive (Levy flight) CTRW is obtained. ${ }^{27}$ In this case, the behavior of the FT of the MP is given by

$$
W(q, t) \simeq e^{-K^{\mu}|q|^{\mu} t},
$$

where $\mu=\rho$ (if $\rho \geq 2, \mu=2$ ). Moreover, $K^{\mu}$ is a generalized diffusion constant. In the case $\alpha<1$ and $\mu<2$, when spatio-temporal coupling of jump length and waiting time are taken into consideration, an $\alpha$ vs $\mu$ phase diagram can be drawn, as reported by Metzler and Klafter. ${ }^{21}$ In the specific case shown here, i.e., mono-d and poly-d micro bead samples, we can expect to detect subdiffusion (i.e., characterized by $\alpha<1$ and $\mu=2$ ) in poly-d samples and Gaussian (i.e., characterized by $\alpha=1$ and $\mu=2$ ) diffusion in mono-d samples, only.

In NMR spectroscopy, the effect of diffusion on an ensemble of $N$ magnetized spins can be described in terms of the behavior of spin phases, $\left[\Phi\left(\mathbf{r}_{i}\right)\right]_{i=1, \ldots . N}$, subjected to an applied magnetic field gradient. ${ }^{6,7}$ The PGSE NMR technique is characterized by radio frequency pulses together with a couple of magnetic field gradient pulses of duration $\delta$, strength $|\mathbf{g}|$, and separated by a delay time $t=\Delta \gg \delta$. The first gradient pulse effectively phase encodes the molecular spins, according to each molecule position. The second decoding gradient pulse completely reverses the spin phase if and only if the molecule has not diffused during the time $\Delta$. Conversely, if the molecule diffuses, the intensity of the signal due to the molecule will be attenuated because of an incomplete phase reverse. In case of ordinary diffusion, the degree of attenuation is a function of both $\mathbf{g}$ and $\Delta$, and occurs at a rate proportional to the self-diffusion coefficient $D$. One of the most interesting features of PGSE is that the measured signal, $E_{\Delta}(\mathbf{k})$, is the FT of the averaged propagator, $\bar{P}_{s}(\mathbf{R}, \Delta)$, i.e., $E_{\Delta}(\mathbf{k}) \propto W(\mathbf{k}, \Delta)$. Since $\Delta$ defines the time window in which the diffusion processes are observed, PGSE NMR techniques allow the measure of effective $\alpha$ and $\mu$ values $\left(\alpha_{\text {eff }}\right.$ and $\left.\mu_{\text {eff }}\right)$ using the aforementioned expressions (1) and (2), respectively. In particular, $\alpha_{\text {eff }}$ is obtained by a fitting procedure of the expression (1) to the experimental data measured by PGSE at different $\Delta$ values. The exponent $\mu_{\text {eff }}$ instead is obtained by a fitting procedure to experimental data at different $\mathbf{q}=1 / 2 \pi \gamma \mathbf{g} \delta$ values, where $\gamma=2.675 \times 10^{8} \mathrm{rad} \mathrm{Hz}$ $\mathrm{T}^{-1}$ is the nuclear gyromagnetic ratio. ${ }^{28}$ Taking into account the $\Delta$ values usually selected to perform PGSE experiments, we expect to measure $\mu_{\text {eff }}=2$ and $\alpha_{\text {eff }} \leq 1$, depending on the investigated heterogeneous sample. However, another peculiar property of the NMR signal in heterogeneous systems 
is its dependence on $\Delta \chi_{m}$ generated at the interface between regions with different $\chi_{m}$. The presence of different susceptibilities can be quantified through $G_{i n t}$ measurements, which can be extracted by the signal decay, generated by a spin-echo (SE) NMR sequence. ${ }^{6,7,29}$ As a consequence, both $\alpha_{\text {eff }}$ and $\mu_{\text {eff }}$ could be, in principle, affected by $\Delta \chi_{m}$.

\section{MATERIALS AND METHODS}

\section{A. Samples}

Eight samples in total were carried out using polystyrene micro-beads (Microbeads AS, Norway) with nominal average diameters of $140,40,30,20,15,10,6$, and $0.050 \mu \mathrm{m}$ and characterized by a $\left|\Delta \chi_{m}\right|=\left|\chi_{m}^{\mathrm{H}_{2} \mathrm{O}}-\chi_{m}^{\text {polystyrene }}\right| \sim 1.59$ $\times 10^{-5}$ in SI units. Six $10 \mathrm{~mm}$ NMR tubes were filled up to a volume of approximately $2 \mathrm{~cm}^{3}$ with beads mono-d in a solution of polyoxyethyle-sorbitan-mono-laurat (Tween 20) $10^{-6} \mathrm{M}$ and deionized water (conductivity $\sim 10^{-6} \Omega^{-1} / \mathrm{cm}$ ), and investigated four months after their preparation. In order to obtain samples characterized by a highly varying multitude of space configurations, which mimic disordered system, three other poly-d specimens were prepared in a similar way, using equal volume fractions of beads of varying sizes. We mixed equal volume fractions of beads of 140,40 , and $6 \mu \mathrm{m}$ to introduce disorder in the samples mainly characterized by $6 \mu \mathrm{m}$ microstructural size. Indeed, by adding microbeads of size larger than $6 \mu \mathrm{m}$ (as an example $140 \mu \mathrm{m}$ and $40 \mu \mathrm{m}$ ), it is possible to obtain a broad distribution of pore sizes around $6 \mu \mathrm{m}$. As a consequence, an increase of effective porosity $p$ is expected in mixed beads samples. The sample, called $140+80+40+10+6 \mu \mathrm{m}$, was made with microbeads of $140,80,40,10$, and $6 \mu \mathrm{m}$, while two other samples were made with micro-beads of 140,40 , and $6 \mu \mathrm{m}$ investigated two months $(140+40+6 \mu \mathrm{m}(2 \mathrm{~m}))$ and one and four months $(140+40+6 \mu \mathrm{m} \quad(1 \mathrm{~m})$ and $140+40+6 \mu \mathrm{m})$ after their preparation, respectively. Finally, one tube filled up with free water was also used as control.

\section{B. Methods}

All measurements were performed on a Bruker $9.4 T$ Avance system, operating with a micro-imaging probe (10 $\mathrm{mm}$ internal diameter bore) and equipped with a gradient unit characterized by a maximum gradient strength of $1200 \mathrm{mT} / \mathrm{m}$, and a rise time of $100 \mu \mathrm{s}$. The temperature of each sample was fixed at $291 \mathrm{~K}$. As a first step, relaxationtime NMR measurements were performed to fully characterize the investigated porous samples. In order to determine the effective porosity $p$, as previously reported, ${ }^{30}$ a SE imaging version using multi slice multi echo sequence (repetition time $T R=1500 \mathrm{~ms}$, matrix $128 \times 128$, slice thickness $S T H=1 \mathrm{~mm}$, in plane pixel dimension $60 \times 60 \mu \mathrm{m}^{2}$, number of scan $N S=8$ ) at various echo times $T E$ (from $2.8 \mathrm{~ms}$ to $300 \mathrm{~ms}$ ) was used to obtain SE decay in different regions of each sample. Conventional mean diffusion coefficient $\bar{D}$ of water in each sample was measured by means of a spectroscopic pulsed g Gradient stimulated echo (PGSTE, TE/TR $=1.8 / 5000 \mathrm{~ms}$, diffusion gradient pulses delay $\Delta=80 \mathrm{~ms}$, diffusion gradient pulses duration $\delta=4.4 \mathrm{~ms}$, and diffusion gradient strength $g$ applied along the $x, y$, and $z$ axes using 48 gradient amplitude steps from 2.6 to $1000 \mathrm{mT} / \mathrm{m})$. $G_{\text {int }}$ along $x, y$, and $z$ axes was measured by using a spectroscopic SE sequence $(T R=1500 \mathrm{~ms}, N S=8)$ with $N=64$ data points (corresponding to 64 echoes refocusing every $2 \mathrm{~ms}$ from 1 to $125 \mathrm{~ms}$ ), as previously described. ${ }^{33}$ Mean value of internal magnetic field gradient $\left(M G_{i n t}\right)$ was obtained as the average value along $x, y$, and $z$ axes. The characteristic diffusion length $\ell_{D}=(2 \bar{D} \Delta)^{1 / 2}$ and the characteristic length $\ell^{*}=\left(\bar{D} / \gamma G_{i n t}\right)^{1 / 3}$, as defined by Sen et al. ${ }^{34}$ and Mitchell et al. ${ }^{29}$ were obtained from these preliminary measurements. In particular, $\ell^{*}$ establishes the spatial region around each bead, within which internal gradients act, dephasing the spins. A spectroscopic PGSTE with $\delta=4.4 \mathrm{~ms}, g=0.12 \mathrm{~T} / \mathrm{m}$ (i.e., $q=22481 \mathrm{~m}^{-1}$ ) along $x, y$, and $z$ axes, with $T R=5000 \mathrm{~ms}$, $N S=32$, and 48 values of $\Delta$ in the range (10/1000) ms was used to collect data either to obtain MSD vs $t$ graphs (following the method described by Angelico et al. ${ }^{35}$ and Scheven et al $^{38}$ ) and to fit Eq. (1) to it, in order to extract the $\alpha_{\text {eff }}$ value along $x, y$, and $z$ axes. Vice versa, a spectroscopic PGSTE with $\Delta / \delta=80 / 4.4 \mathrm{~ms}, T R=5000 \mathrm{~ms}, N S=16$, and 48 gradient amplitude steps from 2.6 to $1000 \mathrm{mT} / \mathrm{m}$ along $x$, $y$, and $z$ axes was used to collect data in order to fit Eq. (2) to it, thus obtaining a measure of the $\mu_{\text {eff }}$ value along $x$, $y$, and $z$ axes. Moreover, in a second step, $\mu$ and $\alpha$ values were also obtained using the BPP-LED PGSTE (bipolar pulse field gradient with eddy current delay) pulse sequence. ${ }^{40}$ The eddy current delay was $5 \mathrm{~ms}$, and all the other sequence parameters were equal to the ones used in PGSTE experiments. The mean values of $\alpha_{\text {eff }}\left(M \alpha_{\text {eff }}\right)$ and $\mu_{\text {eff }}\left(M \mu_{\text {eff }}\right)$ were obtained by averaging $M \alpha_{\text {eff }}=1 / 3 \sum_{i=x, y, z}\left(\alpha_{\text {eff }}\right)_{i}$ and $M \mu_{\text {eff }}=1 / 3 \sum_{i=x, y, z}\left(\mu_{\text {eff }}\right)_{i}$.

All fitting procedures were performed using the Levenberg-Marquardt algorithm.

\section{RESULTS AND DISCUSSION}

\section{A. Preliminary results}

Porosity measurements ${ }^{30}$ underlined that the mean effective porosity, $p$, of all mono-d samples, is approximately equal to $p=0.28 \pm 0.03$. As a consequence, water molecules diffuse in porous systems comprising interconnected pores narrowly distributed around the mean diameter greater than the one of cannon-ball packing configuration, i.e., $\ell_{s}=\sqrt{3}+1 / 4 d$. On the other hand, $140+40+6 \mu \mathrm{m}$ sample is characterized by $p=0.41 \pm 0.03$, while $140+80+40+10+6 \mu \mathrm{m}$ sample is characterized by $p=0.31 \pm 0.03$. Porosity measurements indicate that poly-d samples are more disordered than mono$\mathrm{d}$ ones and that $140+40+6 \mu \mathrm{m}$ is the most disordered sample. In principle, a Gaussian restricted diffusion behavior of water is expected in mono-d samples being characterized by a single typical length scale. Conversely, it is reasonable to expect a subdiffusive anomalous behavior in poly-d samples due to their multiple characteristic length scales on different orders of magnitude. ${ }^{20,31,32}$ In other words, our reasonable physical topic is that a narrow distribution of pore sizes 


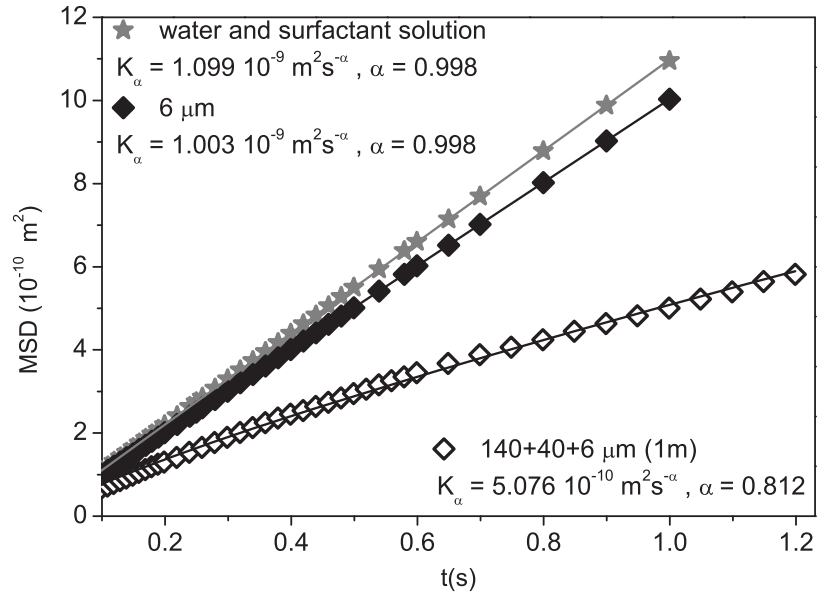

FIG. 1. Plot of MSD as a function of the diffusion time $t=\Delta$ for three different samples: water and surfactant solution, mono-d sample (comprising $6 \mu \mathrm{m}$ beads size) and a poly-d sample (comprising 140, 40, and $6 \mu \mathrm{m}$ beads size). Solid lines represent the curves fit: $M S D(t)=K_{\alpha} t^{\alpha}$. Outcomes of the fit procedure are also displayed for each sample. Star symbol represents proper ordinary diffusion regime.

implies a narrow distribution of residence times (mono-d samples). Vice versa, a broad distribution of pore sizes implies a broad distribution of residence times (poly-d samples). Preliminary results test two hypothesis: (1) AD processes occur in phantoms comprising poly-d polystyrene micro-beads, (2) AD process effects can be observed in data collected with PGSE sequence using $\Delta$ up to $1300 \mathrm{~ms}$ and $g$ strength range from 2.6 to $1000 \mathrm{mT} / \mathrm{m}$. In order to validate these hypothesis, we performed the $M S D$ vs $t=\Delta$ graph shown in Fig. 1, for three different samples: water and surfactant solution, mono-d sample $(6 \mu \mathrm{m})$, characterized by $p$ $=0.28 \pm 0.03$ and poly-d sample $(140+40+6 \mu \mathrm{m})$, characterized by $p=0.41 \pm 0.03$. Solid lines in Fig. 1 represent the curves fit: $M S D(t)=K_{\alpha} t^{\alpha}$. As expected, $M S D$ vs $t$ shows a linear growth in water and surfactant solution and mono-d samples characterized by different slopes. In these cases, the diffusion coefficient, $K_{\alpha=1}=D$, associated with aqueous solution and mono-d samples is $D=1.099 \times 10^{-9} \mathrm{~m}^{2} / \mathrm{s}$ and $D=1.003 \times 10^{-9} \mathrm{~m}^{2} / \mathrm{s}$, respectively. Conversely, a clear departure from linear behavior is found in poly-d sample. These experimental results strongly suggest that an effective $\mathrm{AD}$ occurs in poly-d samples in the range of diffusion times from $100 \mathrm{~ms}$ to $1300 \mathrm{~ms}$. Indeed, by definition, diffusion is anomalous when the variance of molecular displacement increases as a non-unit power of time. In particular, if the waiting time distribution were completely scale-free, e.g., a power-law distribution with diverging mean, the diffusion would be anomalous on all time scales. However, if the waiting time distribution is characterized by a broad distribution on a finite but large range of scales, as presumed in our samples, then the $\mathrm{AD}$ is expected to be restricted to this range of scales and ordinary (Gaussian) diffusion occurs at longer times. This picture is clearly shown in Fig. 1 by the power-law growth of MSD vs $t$ with exponent $\alpha$ smaller than 1 , which is related to the large heterogeneity of typical length scales in such disordered system (poly-d sample). In summary, in Fig. 1 we observe in the poly-d sample an effective $\mathrm{AD}$ which occurs in the diffusion time range (100-
1300) $\mathrm{ms}$ ) determined by the broad distribution of the corresponding pore sizes. Unfortunately, we cannot probe $M S D$ vs $t$ for larger diffusion time $(\Delta>1.5 \mathrm{~s})$ due to $T_{1}$ relaxation time signal loss. This is a limit of the PGSTE NMR technique that hampers to detect MSD vs $t$ asymptotically at long times.

\section{B. Experimental CTRW phase diagram}

The experimental version of the $\alpha$ versus $\mu$ graph suggested by Metzler and Klafter, ${ }^{21}$ for all ten samples investigated, is shown in Fig. 2. Data points obtained from ordered mono-d micro-bead samples (black filled symbols) lie close to the dashed line $\alpha_{\text {eff }}=\mu_{\text {eff }} / 2$, indicating the border line between superdiffusion and subdiffusion regime zones. These points are identified by a similar $M \alpha_{\text {eff }}$ value very close to 1 , and by different values of $M \mu_{\text {eff }}$, ranging from 1.75 to 2, depending on bead sizes. Specifically, data points associated with $0.050 \mu \mathrm{m}, 6 \mu \mathrm{m}$, and $10 \mu \mathrm{m}$ mono-d samples are localized in a subdiffusion regime zone, while $15 \mu \mathrm{m}, 20 \mu \mathrm{m}$, and $30 \mu \mathrm{m}$ ones lie in the superdiffusion regime zone, but all data are close to the border line. The distribution of these data matches the experimental results shown in Fig. 3, where the dynamic feature of water in all mono-d samples is described by the interplay between the characteristic lengths $\ell_{D}, \ell_{s}$, and $\ell^{*} .^{29,34}$ When $\ell_{s}<\ell_{D}, \ell^{*}$ (i.e., bead size lower than $15 \mu \mathrm{m}$ in the investigated samples), motional averaging regime occurs and spins explore the entire pore many times before a dephasing arises, so that any local magnetic field (background gradient) variation is averaged out by diffusion. Conversely, when $\ell^{*}<\ell_{D}, \ell_{s}$ (i.e., bead size is equal to and higher than $15 \mu \mathrm{m}$ in the investigated samples), the slow diffusion case occurs and spins experience a $G_{i n t}$, not totally averaged out, which produces an additional spin dephasing. ${ }^{29}$ Vice versa, data points obtained from disordered poly-d micro-bead samples (empty symbols) lie, as expected, in the subdiffusive region. Specifically, $M \alpha_{\text {eff }}$ decreases as the degree of the disorder increases, while $M \mu_{\text {eff }}$ does not discriminate between

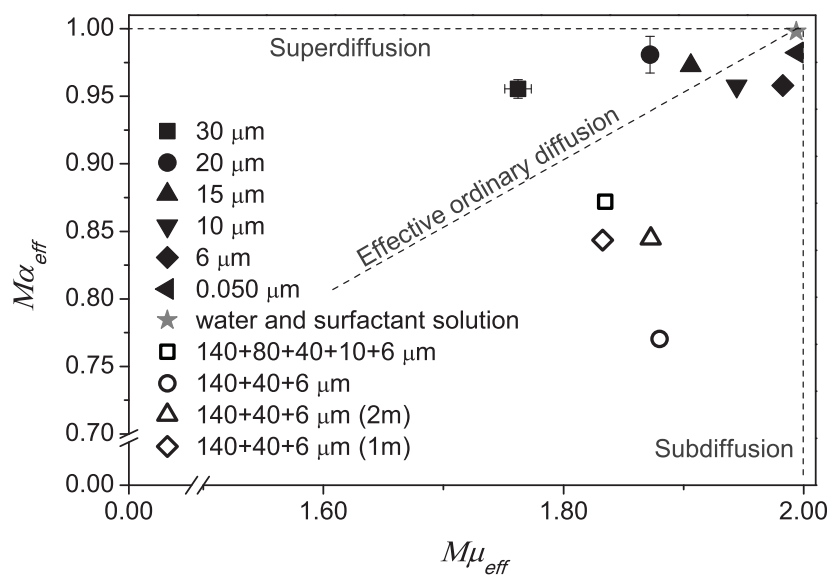

FIG. 2. $M \alpha_{\text {eff }} v s M \mu_{\text {eff }}$ diagram for ordered samples (black filled symbols), disordered samples (empty symbols) and water and surfactant solution (star symbol). Dashed line $2 M \alpha_{\text {eff }}=M \mu_{\text {eff }}$ represents the effective ordinary diffusion region in which $\left\langle x(t)^{2}\right\rangle \propto t^{2 \alpha / \mu}=t$. The two dashed lines $M \alpha_{\text {eff }}=1$ and $M \mu_{\text {eff }}=2$ delimit the regions of superdiffusion $\left(2 M \alpha_{\text {eff }} / M \mu_{\text {eff }}>1\right)$ and subdiffusion $(0<2 M \alpha / M \mu<1)$ regimes. Star symbol location ( $\alpha=1$ and $\mu=2)$ indicates proper ordinary diffusion. 


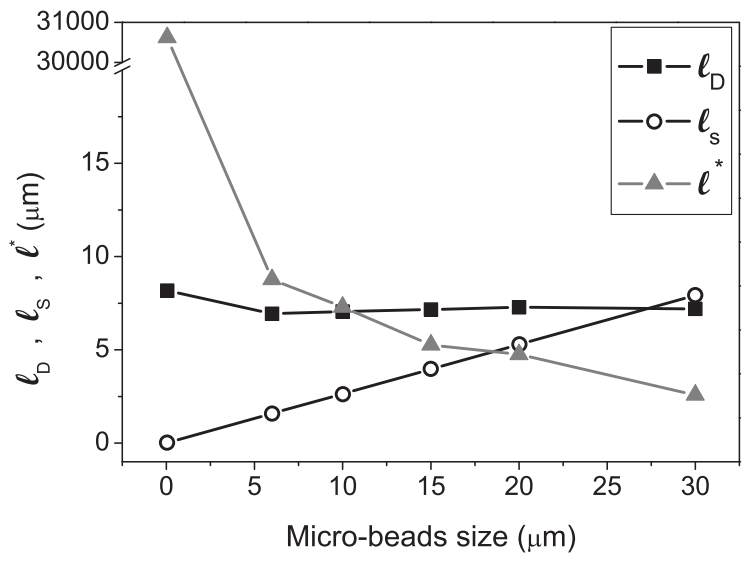

FIG. 3. Characteristic lengths $\ell_{D}, \ell_{s}$, and $\ell^{*}$ as a function of micro-beads size in mono-d samples. This graph shows the dynamic feature of water in the presence of background gradients.

ordered and disordered samples, and assumes values slightly smaller than 2. Water diffusing in investigated poly-d samples has a non-negligible probability to experience broadly distributed resting times in this spatio-temporal window, resulting in an effective anomalous subdiffusive behavior quantified by the decreasing $M \alpha_{\text {eff }}$ value from unity, as system disorder degree increases. Because waiting times depend on pore sizes, number, and thickness of interconnections, a broad distribution in pore diameters (obtainable by mixing beads of different size) is expected to cause a broad distribution in effective "caging" (i.e., resting) times. It is reasonable to suppose that the power law type distribution of waiting times due to the broad distribution of the pore sizes, makes our investigated systems similar to fractal systems, at least in the range of diffusion times (100-1300) ms investigated. Due to the chemical-physical properties of Tween20 surfactant, a possible surface trapping effect of water molecules due to surfactant cannot be excluded. ${ }^{35}$ A natural development of this work will be to measure fractal dimension of the porous system following a procedure similar to the one suggested by Özarslan et $a l .^{5}$ Indeed, our results are similar in form (e.g., $\alpha=2 / d_{w}$, where $d_{w}{ }^{36,37}$ is the fractal dimension of the Brownian motion path of the random walk) to the ones obtained by Özarslan et al. ${ }^{5}$

In order to understand the physical meaning of the $\mu_{\text {eff }}$ parameter, which in principle accounts for an effective superdiffusion process, we guessed that the dependence of $\mu_{\text {eff }}$ on bead size may be spuriously determined by $\Delta \chi_{m}$ at the interface between beads and diffusing water. To validate this assumption, we obtained the plot of Fig. 4, which shows a strong linear correlation $(R=0.996)$ between $M \mu_{\text {eff }}$ and $\ln \left(M G_{i n t}\right)$ for all mono-d samples. In particular, the higher the $M G_{i n t}$ is, the lower the $M \mu_{\text {eff }}$ value is. Conversely, $M \alpha_{e f f}$ does not depend on $\Delta \chi_{m}$ quantified by $M G_{i n t}$, as shown in Fig. 5.

\section{Pseudo-superdiffusion}

In the light of the correlations underlined in Sec. IV B, it is reasonable to assume that $M \mu_{\text {eff }}$ values less than 2 are spurious due to $\Delta \chi_{m}$, that introduces a pseudo-absorb/desorb

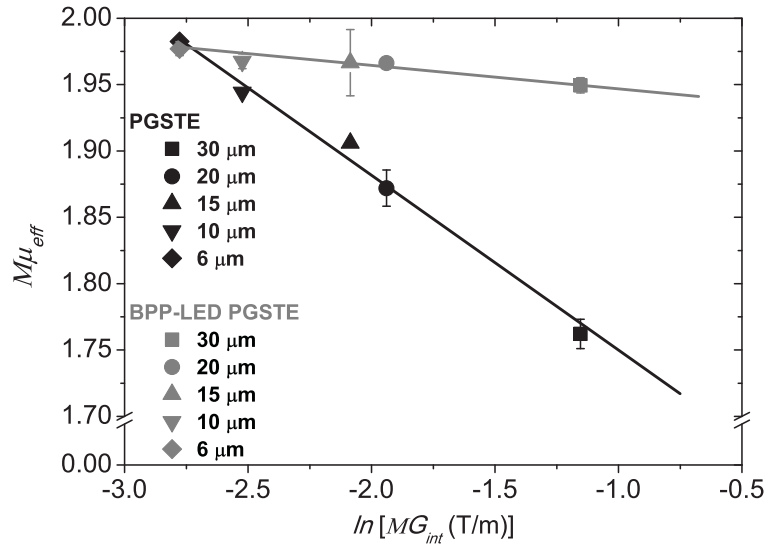

FIG. 4. $M \mu_{\text {eff }}$ as a function of $M G_{i n t}$, measured by PGSTE (black data points) and BPP-LED PGSTE (gray data points) NMR sequences. Black solid line is the regression line $M \mu_{\text {eff }}=(-0.1331 \pm 0.0061) \ln \left(M G_{\text {int }}\right)$ $+(1.613 \pm 0.016), R=0.996$, while gray solid line is the regression line $M \mu_{\text {eff }}=(-0.0148 \pm 0.0031) \quad \ln \left(M G_{\text {int }}\right)+(1.9351 \pm 0.0067)$, $R=0.918$.

process of water molecules at the interface between bead surface and water solution, instead of a real superdiffusion mechanism. This is also shown in Fig. 3, which clearly illustrates that slow diffusion processes become predominant as mean pore diameter $\ell_{s}$ increases. In particular, local gradients impart a phase shift to the spins within $\ell^{*}$ region that adds up to the phase shift given by the diffusion gradient pulse. When background and diffusion gradients are of the same order of magnitude (as in the case of our samples), some spins contribute to increase the degree of PGSE signal attenuation; other spins, which can be in a very distant zone from the first ones, will acquire a phase which will help to increase the signal. Due to indistinguishable spins associated with water molecules, this scenario mimics a superdiffusion regime of water molecules whose signal disappears in one spot and appears in another one. In other words, we suggest that in the investigated micro-bead samples, the measured $\mu_{\text {eff }}$ values less than 2 are only due to an artifact effect generated by $\Delta \chi_{m}$ at the interface between beads and diffusing water. Indeed it is known that water behavior in these kind of interconnected micro-pore systems is Brownian or subdiffusive. ${ }^{23,39}$

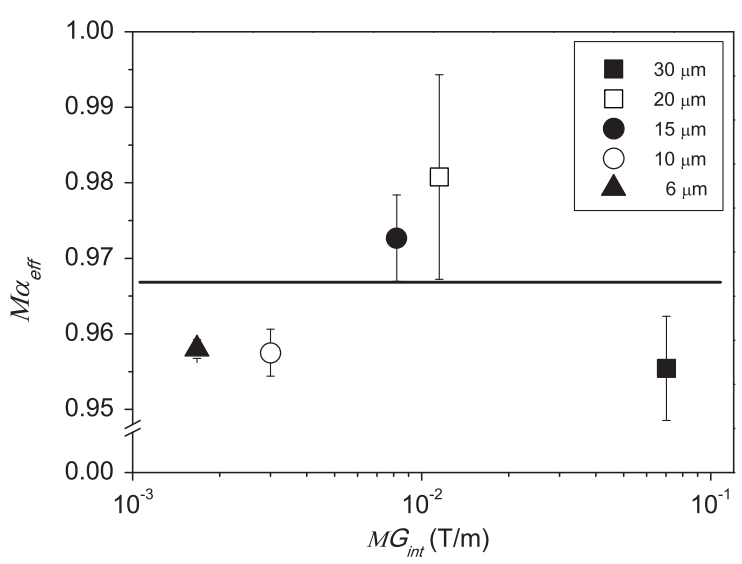

FIG. 5. $M \alpha_{\text {eff }}$ as a function of $M G_{i n t}$, measured by PGSTE NMR sequence. Solid line is the regression line $M \alpha_{\text {eff }}=(0.000 \pm 0.010) M G_{\text {int }}$ $+(0.967 \pm 0.022), R=0.051$. 
However, these artifacts, which simulate superdiffusion, provide information about different pore sizes. It is possible to reduce this spurious pseudo-superdiffusion effects, due to the influence of the coupling between the applied diffusion and internal magnetic field gradients, using bipolar instead of monopolar diffusion gradient pulses. Indeed, it is well known that, unlike monopolar, bipolar composite gradient pulses reduce effects of inhomogeneous background gradients. ${ }^{40}$ In Fig. 4, black data points are obtained using monopolar PGSTE, while gray data points are obtained using bipolar diffusion gradients sequence. As expected, gray data are very close to $M \mu_{\text {eff }}=2$ for all samples. As a consequence, Fig. 4 clearly illustrates that using bipolar diffusion gradients it is possible to eliminate, almost completely, any dependence on internal gradient from $\mu$ values. However, this also means to drastically reduce or eliminate the dependence of $\mu$ on beads size. In the light of these results, in order to study a real superdiffusion process, we suggest to use bipolar sequences, such as background gradient suppression PGSE sequences, recently developed to eliminate the spatially and temporally dependence of $G_{\text {int }}{ }^{41-44}$ Conversely, in micro-structured systems in which anomalous subdiffusive or Gaussian diffusion regimes are expected to be the only possible one, it may be useful to measure $\mu$ without using bipolar gradients to maximize the effect of local $G_{i n t}$ on $\mu$ measurements, in order to effectively discriminate samples characterized by different micro-structural size.

These observations give a basis for further investigations on the correlation between $\mu_{\text {eff }}$ and local $G_{\text {int }}$ to obtain information on the microstructural size features in heterogeneous media. In this contest we have developed a $\mu$-weighted NMR imaging protocol that defines interfaces between substances characterized by different $\Delta \chi_{m}$, better than is currently possible using $T_{2}^{*}$ weighted imaging. In order to stress the importance of results illustrated here, we anticipate that the correlation between $\mu$ and $G_{i n t}$ (that we wanted to highlight using PGSTE sequence without bipolar gradients) suggests that diffusion NMR imaging methods, that rely on collecting the signal decay by varying the gradient strength $g$, are in principle affected by $\Delta \chi_{m}$ artifacts. In particular, our results strongly suggest that the stretched exponential model recently introduced ${ }^{45}$ to quantify the $\mathrm{AD}$ in vivo, is actually based on the quantification of the pseudo-superdiffusion, induced by $\Delta \chi_{m} \cdot{ }^{46-48}$ In our opinion, our work explains some apparent inconsistencies between previous $\mathrm{AD}$ approaches based on $g$ strength varying ${ }^{45,49}$ or on $\Delta$ varying, ${ }^{5}$ allowing a critical revision of previous literature on the $\mathrm{AD}$ applied to biological systems. ${ }^{46}$ Last but not least, the correlation between $\mu$ and local $G_{i n t}$ may help to give more insights into inter-molecular multi-quantum coherences (iMQCs) in liquid systems. ${ }^{50}$ Indeed, it has been demonstrated that iMQCs offer a challenge to investigate porous media. ${ }^{51,52}$ However, their dependence on the interplay between internal gradients and molecular diffusion has yet to be clarified.

\section{CONCLUSION}

In the present paper, the effective CTRW model developed by Metzler and Klafter ${ }^{21}$ was used to experimentally obtain both space and time fractional exponents, $\alpha$ and $\mu$, of water molecules in different porous systems, by using NMR PGSE technique. Magin et al. ${ }^{53}$ also developed a similar approach based on the fractional order differential operators in the Bloch-Torrey equation. ${ }^{53}$ However, Magin's experimental application are limited to space fractional exponent (which Magin called $\beta$, while we call it $\mu$ ) measurement only. In our opinion, as the $\mathrm{AD}$ approach described here does not require any particular a priori knowledge about the geometry and dynamical behavior of the investigated heterogeneous system, it may be a useful tool to probe porous materials and biological tissue microstructural characteristics. Here, we have measured the characteristic parameters of diffusion phase diagram, $\alpha$ and $\mu$, by means of NMR in systems marked by the presence of spatio-temporal competition between effective long rests and apparent long jumps of diffusing molecules. Following the basic common knowledge of complex systems, we made ordered and disordered media using mono- and poly-dispersed micro-beads. We evaluated their disorder degree by measuring the $\alpha$ parameter, which quantifies subdiffusion processes and does not depend on $\Delta \chi_{m}$. Moreover, we highlighted that the real physical mechanism, which gives rise to an apparent superdiffusion, is due to $\Delta \chi_{m}$ between the solid and liquid phase in heterogeneous samples. Due to the peculiar ability of $\mu$ parameter of probing local $\Delta \chi_{m}$ to discriminate different microstructural sizes in heterogeneous media, the development of imaging methods based on $\mu$ contrast may be a new useful tool to study materials and biological tissues. Last but not least, inferring disorder features in heterogeneous media by NMR spectroscopic and imaging techniques may be of paramount value in a variety of applications from oil-well logging and dynamics of polymers to the diagnosis and monitoring in vivo of many diseases in the human body.

${ }^{1}$ P. J. Basser, J. Mattiello, and D. Le Bihan, Biophys. J. 66, 259 (1994).

${ }^{2}$ H. Huang, J. Zhang, H. Jiang, S. Wakana, L. Poetscher, M. I. Miller, P. C. M. Van Zijl, A. E. Hillis, R. Wytik, and S. Mori, Neuroimage 26(1), 195 (2005).

${ }^{3}$ E. O. Stejskal and J. E. Tanner, J. Chem. Phys. 42, 288 (1965).

${ }^{4}$ M. Köpf, C. Corinth, O. Haferkamp, and T. F. Nonnenmacher, Biophys. J. 70, 2950 (1996).

${ }^{5}$ E. Özarslan, P. J. Basser, T. M. Shepherd, P. E. Thelwall, B. C. Vemuri, and S. J. Blackband, J. Magn. Reson. 183(2), 315 (2006).

${ }^{6} \mathrm{P}$. T. Callaghan, Principles of Nuclear Magnetic Resonance Microscopy (Oxford University Press, New York, USA, 1995), pp. 157-169.

${ }^{7}$ W. S. Price, NMR Studies of Translational Motion: Principles and Applications (Cambridge University Press, Cambridge, England, 2009).

${ }^{8}$ P. P. Mitra, P. N. Sen, L. M. Schwartz, and P. Le Doussal, Phys. Rev. Lett. 68(24), 3555 (1992).

${ }^{9}$ S. R. Veith, E. Hughes, G. Vuataz, and S. E. Pratsinis, J. Colloid Interface Sci. 274(1), 216 (2004).

${ }^{10}$ P. T. Callaghan, A. Coy, D. MacGowan, K. J. Packer, and F. O. Zelaya, Nature (London) 351, 467 (1991)

${ }^{11}$ P. T. Callaghan, A. Coy, T. P. J. Halpin, D. MacGowan, K. J. Packer, and F. O. Zelaya, J. Chem. Phys. 97(1), 1 (1992).

${ }^{12}$ E. Özarslan and P. J. Basser, J. Chem. Phys. 128, 154511 (2008).

${ }^{13}$ N. Shemesh, E. Özarslan, P. J. Basser, and Y. Cohen, J. Chem. Phys. 132, 034703 (2010).

${ }^{14}$ D. S. Novikov and V. G. Kiselev, NMR Biomed. 23(7), 682 (2010).

${ }^{15}$ A. Klemm, R. Metzler, and R. Kimmich, Phys. Rev. E 65, 021112 (2002).

${ }^{16}$ T. H. Solomon, E. R. Weeks, and H. L. Swinney, Phys. Rev. Lett. 71(24), 3975 (1993).

${ }^{17}$ E. Fischer, R. Kimmich, U. Beginn, M. Möller, and N. Fatkullin, Phys. Rev. E 59, 4079 (1999). 
${ }^{18}$ V. Plerou, P. Gopikrishnan, L. A. N. Amaral, X. Gabaix, and H. E. Stanley, Phys. Rev. E 62(3A), R3023 (2000).

${ }^{19}$ G. Seisenberger, M. U. Ried, T. Endreß, H. Büning, M. Hallek, and C. Bräuchle, Science 294(5548), 1929 (2001).

${ }^{20}$ A. Cortis, C. Youjian, H. Scher, and B. Berkowitz, Phys. Rev. E 70, 041108 (2004).

${ }^{21}$ R. Metzler and J. Klafter, Phys. Rep. 339, 1 (2000).

${ }^{22}$ W. R. Schneider and W. Wyss, J. Math. Phys. 30, 134 (1989).

${ }^{23}$ A. Zoia, Phys. Rev. E 77, 04115 (2008).

${ }^{24}$ N. Periasamy and A. S. Verkman, Biophys. J. 75, 557 (1998).

${ }^{25}$ X. L. Wu and A. Libchaber, Phys. Rev. Lett. 84(13), 3017 (2000).

${ }^{26}$ A. T. Watson and C. T. P. Chang, Prog. Nucl. Magn. Reson. Spectrosc. 31, 343 (1997).

${ }^{27}$ A. Gabrielli and F. Cecconi, J. Stat. Mech.: Theory Exp. 10, 10007 (2007).

${ }^{28}$ P. T. Callaghan, Principles of Nuclear Magnetic Resonance Microscopy (Oxford University Press, New York, USA, 1995), p. 28.

${ }^{29}$ J. Mitchell, T. C. Chandrasekera, M. L. Johns, L. F. Gladden, and E. J. Fordham, Phys. Rev. E 81, 026101 (2010).

${ }^{30}$ T. Amitay-Rosen, A. Cortis, and B. Berkowitz, Environ. Sci. Technol. 39, 7208 (2005).

${ }^{31}$ B. Berkowitz and C. Braester, Water Resour. Res. 27(12), 3159 (1991).

${ }^{32}$ B. Berkowitz and D. P. Hansen, Transp. Porous Media 45, 303 (2001).

${ }^{33}$ S. De Santis, M. Rebuzzi, G. Di Pietro, F. Fasano, B. Maraviglia, and S. Capuani, Phys. Med. Biol. 55(19), 5767 (2010).

${ }^{34}$ P. N. Sen, A. André, and A. Axelrod, J. Chem. Phys. 111(14), 6548 (1999).

${ }^{35}$ R. Angelico, A. Ceglie, U. Olsson, G. Palazzo, and L. Ambrosone, Phys. Rev. E 74, 031401 (2006).

${ }^{36} \mathrm{D}$. ben-Avraham and S. Havlin, Diffusion and Reactions in Fractals and Disordered Systems (Cambridge University Press, Cambridge, UK, 2000).

${ }^{37}$ S. Havlin and D. ben-Avraham, Adv. Phys. 51(1), 187 (2002).
${ }^{38}$ U. M. Scheven, J. P. Crawshaw, V. J. Anderson, R. Harris, M. L. Johns, and L. F. Gladden, Magn. Reson. Imaging 25, 513 (2007).

${ }^{39}$ M. R. Horton, F. Höfling, J. O. Rädler, and T. Franosch, Soft Matter 6, 2648 (2010).

${ }^{40}$ D. H. Wu, A. D. Chen, and C. S. Johnson, J. Magn. Reson. A 115, 260 (1995).

${ }^{41}$ J. G. Seland, G. H. Sorland, K. Zick, and B. Hafskjold, J. Magn. Reson. 146, 14 (2000).

${ }^{42}$ P. Galvosas, F. Stallmach, and J. Kärger, J. Magn. Reson. 166, 164 (2004).

${ }^{43}$ P. Z. Sun, J. Magn. Reson. 187, 177 (2007).

${ }^{44}$ G. Zheng and W. S. Price, J. Magn. Reson. 195, 40 (2008).

${ }^{45}$ K. M. Bennett, K. M. Schmainda, R. Bennett, D. B. Rowe, H. Lu, and J. S. Hyde, Magn. Reson. Med. 50, 727 (2003).

${ }^{46}$ S. Capuani, M. Palombo, S. De Santis, and A. Gabrielli, in Proceedings of the 19th Scientific Meeting of the International Society for Magnetic Resonance in Medicine, Montreal, Quebec, 7-13 May, 2011.

${ }^{47}$ M. Palombo, S. De Santis, and S. Capuani, in Proceedings of the 19th Scientific Meeting of the International Society for Magnetic Resonance in Medicine, Montreal, Quebec, 7-13 May, 2011.

${ }^{48}$ S. De Santis, A. Gabrielli, E. Macaluso, M. Bozzali, and S. Capuani, in Proceedings of the 19th Scientific Meeting of the International Society for Magnetic Resonance in Medicine Montreal, Quebec, 7-13 May, 2011.

${ }^{49}$ S. De Santis, A. Gabrielli, M. Bozzali, B. Maraviglia, E. Macaluso, and S. Capuani, Magn. Reson. Med. 65(4), 1043 (2011).

${ }^{50}$ W. Richter, S. Lee, W. S. Warren, and Q. He, Science 267(5198), 654 (1995).

${ }^{51}$ S. Capuani, M. Alesiani, F. M. Alessandri, and B. Maraviglia, Magn. Reson. Imaging 19(3-4), 319 (2001).

${ }^{52}$ F. M. Alessandri, S. Capuani, and B. Maraviglia, J. Magn. Reson. 156, 72 (2002).

${ }^{53}$ R. L. Magin, O. Abdullah, D. Baleanu, and X. J. Zhou, J. Magn. Reson. 190, 255 (2008). 